\title{
Perspectivas universalistas y relativistas de la lectura y la escritura en dos casos en Colombia: una mirada antropológica*
}

\author{
Daniel Rudas Burgos* \\ Andrey Castiblanco ${ }^{* * *}$
}

Recibido: 28 de junio de 2017 - Aprobado: 1 de octubre de 2017

\section{Resumen}

A partir de la descripción de una selección de casos etnográficos, este artículo plantea que hay dos perspectivas para entender la lectura y la escritura como prácticas sociales: una universalista y otra relativista. La universalista supone que la escritura tiene efectos cognitivos y culturales per se, y que sus formas pueden generalizarse partiendo de la tradición occidental; mientras que la relativista supone que la escritura adquiere sentido en conexión con los contextos locales y sus formas dependen de eventos particulares. En el presente artículo, ambas perspectivas son utilizadas para interpretar dos casos observados en Colombia: un programa de alfabetización para adultos en El Codito (un barrio periférico de Bogotá), en el cual se adaptó el currículo a la perspectiva relativista, y un club de lectura para jóvenes en Guatavita (un municipio cercano a la misma ciudad), que estuvo basado en pedagogías dialógicas y críticas. Se concluye que las perspectivas universalistas y relativistas coexisten en ambos programas.

Palabras clave: alfabetización, antropología de la escritura, eventos de escritura, educación extraescolar, pedagogía crítica.

Artículo de investigación. Resultado del proyecto "Prácticas de escritura y lectura en Cundinamarca", financiado por el Instituto Caro y Cuervo. Grupo de Investigación en Lingüística, línea Lenguaje, educación y diversidad. DOI: http:// dx.doi.org/10.15332/ s0120-8454.2018.0092.01

** Antropólogo y Magíster en Educación. Investigador del Instituto Caro y Cuervo, Bogotá. Estudiante de doctorado en Antropología y Educación del Teachers College, Columbia University, Nueva York. Dirección postal: Calle 10 \# 4-69, Bogotá. Correo electrónico: daniel.rudas-burgos@tc.columbia.edu. Orcid: http://orcid.org/0000-0002-4662-8528

*** Antropólogo de la Pontificia Universidad Javeriana, Bogotá. Investigador del Instituto Caro y Cuervo. Líder de lectoescritura del programa de alfabetización de ColombiaCrece. Dirección postal: Calle 10 \# 4-69, Bogotá. Correo electrónico: andrey.castiblanco@javeriana.edu.co. Orcid: http://orcid.org/0000-0002-0868-7594 


\title{
Universalistic and relativistic perspectives of literacy in two cases in Colombia: An anthropological view*
}

\author{
Daniel Rudas Burgos** \\ Andrey Castiblanco***
}

\section{Abstract}

Based on a description of a selection of ethnographical cases, this article states that there are two perspectives for understanding literacy as a social practice: Universalistic and relativistic. Universalistic perspective assumes that literacy has by itself cognitive and cultural effects, and that it is possible to generalize its forms, taking as a starting point the Western tradition. In contrast, relativistic perspective assumes that literacy acquires its meaning in connection with local contexts, and its forms depend on particular events. In this paper, both perspectives are used for interpreting two cases observed in Colombia: First, an adult literacy program in El Codito (a peripheral neighborhood in Bogota) in which the curriculum was adapted to the relativistic perspective; second, a reading club for young people in Guatavita (a village close to the same city) based on dialogical and critical pedagogies. At the end, it is argued that universalistic and relativistic perspectives coexist in both of the observed cases.

Keywords: literacy, anthropology of literacy, literacy event, out-of-school education, critical pedagogies

This paper is the final result of the project "Literacy practices in Cundinamarca," funded by the Caro y Cuervo Institute. The project is part of the Research Group in Lingüistics, especifically the Language, Education and Divesity research line. DOI: http://dx.doi.org/10.15332/ s0120-8454.2018.0092.01

* BA in Anthropology, MA in Education. Research associate at Caro y Cuervo Institute. Ph, D. student at the Anthropology and Education Program at Teachers College, Columbia University. Postal address: Calle 10 \# 4-69, Bogotá. E-mail: daniel.rudas-burgos@tc.columbia.edu. Orcid: http://orcid.org/0000-0002-4662-8528

..* BA in Anthropology at Pontificia Universidad Javeriana in Bogotá. Research assistant at Caro y Cuervo Institute. Reading and Writing leader at the ColombiaCrece literacy program. Postal address: Calle 10 \# 4-69. E-Mail: andrey.castiblanco@ javeriana.edu. Orcid: http://orcid.org/0000-0002-0868-7594 


\title{
Perspectives universalistes et relativistes de la lecture et de l'écriture en Colombie: un regard anthropologique sur deux cas d'étude*
}

\author{
Daniel Rudas Burgos \\ Andrey Castiblanco***
}

\section{Résumé}

Par le biais d'une description ethnographique, cet article propose deux perspectives d'analyse de la lecture et de l'écriture en tant que pratiques sociales : l'une, universaliste ; l'autre, relativiste. La première assume que l'écriture a des effets cognitifs et culturels per se et qu'elle peut être instaurée partout selon le modèle occidental. La seconde, quant à elle, entend l'écriture par rapport aux contextes locaux qui lui donnent un sens particulier. Cet article fait appel à ces perspectives afin d'interpréter deux cas observés en Colombie : un programme d'alphabétisation pour les adultes d'un quartier défavorisé de Bogotá (El Codito) et un club de lecture pour les jeunes d'un village près de Bogotá (Guatavita). On peut conclure que ces deux perspectives, universaliste et relativiste, coexistent dans les deux programmes analysés.

Mots clés : alphabétisation, anthropologie de l'écriture, actes d'écriture, éducation extra-scolaire, pédagogie critique.

Cet article s'inscrit dans le cadre d'une recherche intitulée "Lecture et écriture en tant que pratiques culturelles ", développé au sein de l'Instituto Caro y Cuervo. Groupe de recherche en Linguistique l'Instituto Caro y Cuervo. DOI: http://dx.doi.org/10.15332/ s0120-8454.2018.0092.01

" Licencié en Anthropologie et Master en Éducation. Investigador del Instituto Caro y Cuervo, Bogotá. Doctorant en Anthropologie et Éducation au Teachers College, Columbia University, Nueva York. Adresse postale : Calle 10 \# 4-69, Bogotá. Mail : daniel.rudas-burgos@tc.columbia.edu. Orcid: http://orcid.org/0000-0002-4662-8528

... Licencié en anthropologie, Pontificia Universidad Javeriana, Bogotá. Chercheur à l'Instituto Caro y Cuervo. Adresse postale : Calle 10 \# 4-69, Bogotá. Mail : andrey.castiblanco@javeriana.edu.co. Orcid: http://orcid.org/0000-0002-0868-7594 


\section{Introducción}

Los estudios en lectura y escritura han pasado de un modelo universalista, autónomo y unitario, que suponía que la escritura producía en sí misma algún tipo de efecto (por ejemplo, pensamiento crítico o abstracto), a un modelo más sensible a la diversidad cultural, que tiene en cuenta los intereses y necesidades de cada grupo social. Esta transición fue señalada por Corrine Wickens y Jennifer Sandlin (2007) como parte de su crítica a las políticas públicas internacionales en lectura y escritura. En efecto, las autoras aplicaron técnicas de análisis crítico del discurso y teoría fundamentada a varios textos publicados por la Unesco y el Banco Mundial en la primera década del siglo XXI y hallaron que, a pesar de que prevalece una ideología neocolonial en ambos documentos, particularmente la Unesco ha pasado de un modelo universalista a otro más relativista.

En este artículo sostendremos, de acuerdo con la literatura existente, que las investigaciones antropológicas acerca de la lectura y la escritura han tenido una transición semejante, porque pasaron a su vez de un modelo etnocéntrico y determinista, preocupado por los beneficios que traía la escritura occidental cuando la adoptaban comunidades sin escritura, a un modelo relativista, que ha buscado formas particulares de dar sentido a la lectura y la escritura en contextos situados (Bartlett, López, Vasudevan y Warriner, 2011; Barton y Hamilton, 2000; Roberts y Street, 2013; Street, 2013). De la misma manera, sostendremos que, cuando se aplican en la práctica estas ideas, ambas perspectivas coexisten (Papen, 2005; Rogers, 1999).

Este artículo está organizado en dos grandes secciones. En la primera haremos un recorrido por los aportes de la antropología a los estudios en lectura y escritura y en la segunda discutiremos dos casos observados en Colombia: el primero es un programa de alfabetización para adultos que se realizó en un barrio periférico de Bogotá y el segundo es un club de lectura informal para jóvenes que se organizó en un municipio cercano a la misma ciudad.

Es importante señalar que la antropología opera a través de la interpretación de etnografías, esto es, de casos concretos observados en diferentes grupos sociales. En consecuencia, para ilustrar los aportes de la disciplina a los estudios sobre lectura y escritura haremos un recorrido histórico por varias etnografías enfocadas específicamente en el tema. Nuestra pretensión para este artículo, sin embargo, no es hacer un estudio exhaustivo, sino dar un panorama general de estos aportes para efectos de ilustrar la transición mencionada.

Para los dos casos observados, organizaremos la discusión alrededor de las relaciones que estos han tenido con las perspectivas universalista y relativista de la lectura y la escritura. Al final concluiremos que en los dos casos ambas perspectivas coexisten. 


\section{Antropología de la lectura y la escritura: de un modelo universalista a uno relativista}

En antropología, los estudios sobre lectura y escritura engloban lo que en inglés cabe bajo el término literacy, que se refiere a la enseñanza escolar del lenguaje escrito; los procesos de alfabetización para adultos y la lectura y escritura vistas como habilidades o como prácticas ${ }^{1}$. En estos estudios se asume que leer y escribir son prácticas situadas en contextos particulares. Así, para David Barton y Mary Hamilton, el objeto de los estudios antropológicos en lectura y escritura incluye "los modos generales y culturalmente establecidos de utilizar el lenguaje escrito, de los cuales las personas echan mano en sus vidas" (2000, p. 7, traducción nuestra)2 ${ }^{2}$, y para los antropólogos Allan Luke y Peter Freebody:

Leer es una práctica social en la que se usa el texto escrito como un medio para construir y reconstruir afirmaciones, mensajes y significados. Leer realmente "se hace" en espacios públicos y privados en el día a día de las instituciones comunitarias, laborales y académicas. (1997, p. 185, traducción nuestra) ${ }^{3}$

Como práctica social, todo lo asociado con leer y escribir ha sido estudiado en la antropología a través casos específicos, a partir de los cuales se han derivado los aportes de la disciplina. De acuerdo con Brian Street (1993), es posible distinguir dos perspectivas diferentes entre los antropólogos a la hora de construir estos aportes: la perspectiva autónoma y la perspectiva ideológica. Ambas tienen como fuente la práctica, pero se diferencian en que la autónoma estudia principalmente la escritura occidental y sus análisis son universalizantes, mientras que la ideológica estudia otras formas de escritura y sus análisis son relativistas.

La perspectiva autónoma (universalista) trata de mostrar los efectos universales que tiene el alfabeto en la humanidad y afirma que el uso de este produce en sí mismo (de forma autónoma) efectos cognitivos y culturales. La perspectiva ideológica (relativista), en cambio, considera que la escritura no tiene efectos en sí misma, sino que estos son relativos a cada contexto particular. En esta perspectiva, los discursos, actitudes y relaciones de poder (ideologías) tienen un papel preponderante.

1 Seguramente el uso de un término tan amplio, proveniente del inglés, se debe a que hay muchos estudios sobre el tema hechos desde la antropología anglosajona. Literacy se podría traducir como "alfabetización", para referirse a la enseñanza de la lectura, particularmente en adultos; como "lectoescritura", para referirse a las habilidades y competencias lectoras y escritoras, particularmente en niños; o como "cultura escrita", para aludir al impacto del lenguaje escrito en una sociedad. Según la Unesco (2005), literacy puede referirse a las habilidades de leer y escribir, a la práctica de esas habilidades, al proceso de aprendizaje de estas o al análisis discursivo de los textos producidos.

2 En el original: "The general cultural ways of utilizing written language which people draw upon their lives".

3 En el original: "Reading is a social practice using written text as a means of the construction and reconstruction of statements, messages, and meanings. Reading is actually "done" in the public and private spaces of everyday community, occupational and academic institutions". 
El antropólogo más representativo de la perspectiva universalista es Jack Goody. Él hizo un extenso trabajo de campo entre los lodagaa del noroccidente de Ghana $\mathrm{y}$, entre otras cosas, pasó por escrito algunos relatos míticos de esa sociedad. En su opinión (Goody, 2000; Goody y Watt, 1996), el hecho de introducir la escritura tuvo un efecto en el pensamiento y la organización política de los lodagaa. La versión oral de los mitos que contaban las autoridades tradicionales variaba según el contexto, pero con el texto escrito se creó una versión canónica que podía ser contrastada con las versiones orales. Ese contraste le permitió a los lodagaa ser críticos con respecto a sus propios mitos. Para Goody, esto podría ser el germen del pensamiento crítico entre los lodagaa e incluso la puerta de entrada al pensamiento lógico-científico occidental.

Las investigaciones de Goody estuvieron relacionadas con el trabajo de Walter Ong (1987). Según este autor, la escritura se diferencia de la oralidad en que esta ocurre en situaciones concretas en las que hay contacto inmediato entre los interlocutores, mientras que aquella ocurre en un soporte gráfico que separa espacial y temporalmente a los interlocutores. Según el autor, esta separación tiene como consecuencia que quien escribe debe tener en cuenta la multiplicidad de situaciones en las puedan ser recibidos sus mensajes, mientras que quien habla solamente debe tener en cuenta la situación inmediata en la que los suyos en efecto se reciben. Para Ong, esta característica de la escritura genera una serie de cambios en el pensamiento humano: mientras la forma de pensar propia de lo oral es concreta, circunstancial y fuertemente vinculada al presente, la forma de pensar propia de lo escrito es abstracta, universal y vinculada al pasado o al futuro, lo cual es condición necesaria para el surgimiento de la literatura.

En contraste, los estudiosos de la perspectiva relativista argumentan que los universalistas hacen que ciertas prácticas de lectura y escritura se vuelvan invisibles, impensables o sean valoradas como deficientes. Así por ejemplo, Bryan Maddox (2005) describe a un grupo de mujeres en Bangladesh que usa la lectura del Corán en árabe, aun sin entender la lengua, para efectos de aliviar las tensiones y la ansiedad que provoca la pobreza en sus familias. A su vez, estas mujeres usan la escritura en su lengua natal para llevar registro de la economía doméstica y ayudar de esta manera a reducir los riesgos propios de su situación económica. De acuerdo con Maddox, estos dos usos del lenguaje escrito suelen no ser tenidos en cuenta puesto que no encajan en los supuestos del modelo autónomo (crear pensamiento crítico o abstracto), aunque se acerquen a las expectativas de inclusión económica de organizaciones internacionales como la Unesco o el Banco Mundial.

Uno de los ejemplos más citados para criticar la perspectiva universalista es el trabajo de Harold Conklin (1949), quien describió el sistema de escritura de los mindoro en Filipinas y su lugar en esa sociedad. Se trataba de un silabario que se ejecutaba haciendo incisiones con un cuchillo en cañas de bambú. Este sistema de escritura era usado fundamentalmente para hacer poemas de amor. Según Conklin, los jóvenes mindoro que buscaban pareja debían convencer a los mayores de enseñarles a escribir los poemas en el bambú para poder participar 
de los ritos de matrimonio. La escritura en este caso estaba fuertemente vinculada con las tradiciones mindoro y no producía los textos canónicos ni el pensamiento crítico que postula Goody.

Otro caso que cuestiona la perspectiva universalista lo describió el antropólogo Charles Ferguson (1987). Él reconstruyó la historia de un grupo de misioneros alemanes que intentaron introducir la escritura occidental entre los diyari en Australia al final del siglo XIX. Además de evangelizar, los misioneros levantaron una escuela para enseñar a los diyari a leer y escribir en su vida cotidiana, tal como lo hacían ellos mismos: escribiendo cartas, llevando diarios, registrando eventos o llevando cuentas. Según narra Ferguson, muchos diyari aprendieron a usar la escritura de todas esas maneras, pero cuando la misión terminó, con la llegada del poder británico a inicios del siglo $\mathrm{XX}$, solo unos pocos continuaron escribiendo, y los que lo hicieron se limitaron a escribir cartas a los misioneros con quienes habían trabado amistad. Para Ferguson, esto desmiente que la escritura tenga en sí misma algún efecto permanente en las sociedades humanas.

Por otra parte, Niko Besnier (1988) probó que la tesis de Ong, según la cual la escritura está asociada al pensamiento abstracto mientras que la oralidad lo está al pensamiento concreto, es una equivocación. Besnier estudió la escritura entre los nukulaelae de Tuvalú. Ellos adquirieron el lenguaje escrito de los misioneros cristianos en el siglo XIX y lo usaban tanto para los servicios religiosos como para la escritura de cartas. Besnier recolectó muestras de ese tipo de textos, así como de expresiones orales relacionadas; después, transcribió todo el material y lo segmentó en unidades más pequeñas que podían relacionarse lingüísticamente o bien con el pensamiento abstracto o bien con el pensamiento concreto. Mediante un sofisticado análisis factorial, el investigador encontró que la aparición de unidades relacionadas con el pensamiento abstracto no tenía una correlación fuerte con la escritura, sino que en cambio estaba relacionada con la formalidad de los contextos en los que aparecía. Con este estudio, la relación causal entre pensamiento abstracto y escritura quedó en entredicho.

Además, quienes estudian la lectura y la escritura desde la perspectiva relativista piensan que una visión universalista conduce a ciertas formas de discriminación, porque parte del principio de que la oralidad y la escritura están separadas. Según Street (2009), la oposición entre oralidad y escritura no existe en la práctica y los efectos que supuestamente causa la escritura o bien ya existían en la cultura oral o bien fueron introducidos por instituciones como la escuela, pero de todas maneras no son atribuibles a la escritura en sí misma. De forma similar, para Celia Roberts y Brian Street (2013) el efecto no intencionado que tiene la oposición entre oralidad y escritura es que la primera se considera sinónimo de ignorancia mientras la segunda se considera sinónimo de conocimiento, con lo cual los grupos humanos que no practican la escritura terminan siendo etiquetados como ignorantes y sus saberes tradicionales resultan, por lo tanto, anulados.

En esa misma línea, Marcia Farr (2009) y Uta Papen (2001) argumentan que los beneficios universales que se le otorgan a la escritura han creado un discurso del 
déficit que cae sobre las personas que no tienen una "adecuada alfabetización", lo que tiene, por supuesto, efectos concretos en sus realidades. Por ejemplo, Farr (2009) hace su trabajo en una comunidad transnacional de mexicanos en Chicago y Michoacán, donde las personas escriben de forma "lírica": ellos dicen que escriben como hablan, lo que se diferencia de la norma estándar, que promueve una sola forma de escribir, la escolar ortográfica. La escritura lírica suelen usarla en su correspondencia. A pesar de que esta escritura funciona entre ellos, algunos michoacanos que escriben de forma lírica afirman que no saben leer y escribir, así que desconocen sus prácticas locales. Por eso, a la hora de conseguir un trabajo y enfrentar una evaluación estandarizada de alfabetización, son clasificados como analfabetas, lo que les impide ser contratados. De este modo, Farr muestra que cualquier definición de alfabetización trae consigo un poder ideológico que sitúa a la gente en relaciones jerárquicas que crean determinadas realidades sociales y tienen efectos materiales reales.

Otro ejemplo contundente lo ofrece Bernard Lahire, quien muestra cómo los relatos orales y escritos de niños alfabetizados pertenecientes a sectores populares urbanos de Francia son estigmatizados en la escuela porque "no están construidos como pequeños universos autónomos de sentido" (2006, p. 144).

Con el fin de evitar postulados universalizantes, la perspectiva relativista, basada en la idea de que la lectura y la escritura son prácticas social e históricamente construidas, ha ideado conceptos que permiten describir las formas en las que leer y escribir cobran sentido en contextos situados. Uno de los más importantes es el de evento de lectura y escritura (literacy event). Shirley Heath (2009) propuso este concepto basada en una etnografía de larga duración enfocada en el uso del lenguaje escrito de los pobladores de un asentamiento industrial de Carolina del Norte, en Estados Unidos. Uno de los casos más notables que narra esta etnografía es el de la lectura y escritura de cartas en una comunidad de trabajadores afroamericanos. Lejos de haber sido un acto individual, la lectura de cartas era un evento colectivo en el que intervenía la oralidad. De la misma manera, la oralidad era usada para componer colectivamente las respuestas.

A partir de varias observaciones semejantes en las que la frontera entre oralidad y escritura no está claramente demarcada en la práctica, Heath propuso el concepto de evento de lectura y escritura y lo definió como "las ocasiones en las que el lenguaje escrito está integrado a la naturaleza de las interacciones de los participantes y a sus procesos y estrategias de interpretación" (Heath, 2009, p. 50 , traducción nuestra $)^{4}$. Para ello se basó en la etnografía de la comunicación de Dell Hymes (1986). El mencionado concepto, por su relación con la etnografía de la comunicación, pone el énfasis en la interacción y permite observar las prácticas de lectura y escritura en el contexto sociohistórico de las prácticas culturales y de las instituciones que las producen (Marinho, 2009).

4 En el original: "Occasions in which written language is integral to the nature of participants' interactions and their interpretive processes and strategies". 
Dicho concepto, además, ha sido muy utilizado en las investigaciones más recientes sobre lectura y escritura. Por ejemplo, Judith Kalman (2003) describe cómo en una plaza de Ciudad de México hay escribanos que venden sus servicios de mecanografía a los ciudadanos que necesitan hacer trámites administrativos. Para ella, el encuentro entre los escribanos y sus clientes es un evento de lectura y escritura, en tanto que el lenguaje escrito está integrado a la interacción. Kalman argumenta que esta interacción tiene consecuencias importantes para todos los involucrados, porque el texto resultante puede ser útil o no para el trámite que se vaya a hacer, y el prestigio de los escribanos depende de ello.

Más recientemente, ha habido un esfuerzo por aplicar las investigaciones antropológicas en lectura y escritura a programas de alfabetización, especialmente para adultos. Alan Rogers (1999) propuso una metodología llamada aproximación a las lecturas y escrituras reales (real literacies approach), en el que tanto los objetivos del proceso de alfabetización como los textos mismos en los que se basa son identificados por los facilitadores a través de un proceso de investigación etnográfica. Por ejemplo, Brian Street en compañía de Alan Rogers y David Baker (2006) implementaron un programa de alfabetización que usaba esa metodología en Nirantar, India. Efectivamente consiguieron que los facilitadores identificaran prácticas de escritura relevantes para los habitantes del lugar.

Uno de los hallazgos más interesantes de estas aplicaciones prácticas ha sido constatar que en muchas comunidades la relevancia del aprendizaje de la lectura y la escritura está asociada al prestigio de la educación escolarizada. A partir de su experiencia práctica en alfabetización para adultos en Namibia, Uta Papen (2005) sostiene que es frecuente que las poblaciones locales estén interesadas en recibir una educación tradicional, con las características de una perspectiva universalista, como un fin en sí mismo. Frente a esta situación, Rogers (1999) ha planteado que se debe partir de la perspectiva universalista, pero introduciendo paulatinamente una perspectiva relativista que reconozca los intereses y expectativas de las poblaciones locales y se base en los hallazgos etnográficos que se hagan junto con los facilitadores, que deben hacer parte de la población local.

\section{Dos etnografías en Colombia}

Los autores de este artículo hacemos parte de una línea de investigación, recientemente creada en el Instituto Caro y Cuervo ${ }^{5}$, llamada Lenguaje, educación y diversidad. Las dos etnografías que presentamos son producto de un proyecto de investigación titulado "Prácticas no convencionales de lectura y escritura en Cundinamarca". Ambos investigadores adoptamos la perspectiva relativista al iniciar el estudio. Ello implicó el uso de una aproximación etnográfica, esto es, el contacto directo con las realidades sociales estudiadas, con una actitud de extrañamiento, en la que nos permitimos poner en duda nuestros propios

5 El Instituto Caro y Cuervo es una entidad pública adscrita al Ministerio de Cultura de Colombia, que se enfoca en temas de lingüística y literatura en el país (www.caroycuervo.gov.co). 
preconceptos (Velasco y Díaz de Rada, 2006), para poder dar cuenta y razón de los eventos de lectura y escritura tal como los viven las personas que los realizan - los literacy events planteados por Heath (2009)-.

En los dos casos estudiados, los investigadores participamos activamente, tanto en calidad de instructores como en condición de colaboradores. Las etnografías fueron hechas por medio de observación participante, conversaciones informales y entrevistas semiestructuradas.

\section{ColombiaCrece ${ }^{6}$}

ColombiaCrece es una fundación que tiene programas de alfabetización y validación en tres sectores del norte de Bogotá: Prado, Suba y El Codito. Esta investigación se realizó más intensamente en El Codito, un sector periférico de la ciudad que se formó en los años setenta cuando una gran ola de inmigrantes de las zonas rurales del país llegó a Bogotá escapando de la violencia o buscando mejores oportunidades laborales. El programa de alfabetización se creó en 2010, un año después de la creación del de validación del bachillerato, ya que muchas de las personas que querían validar no sabían leer ni escribir, lo que impedía que asistieran a las clases.

En este programa de alfabetización se enseñan lectura, escritura, matemáticas y arte, principalmente, a adultos mayores de El Codito ${ }^{7}$. Las clases las dan voluntarios, en su mayoría jóvenes universitarios, los sábados de 7:30 a. m. a 12:30 p. m. Los contenidos del programa fueron creados por estos voluntarios "intuitivamente", esto es, sin ningún entrenamiento formal en educación o alfabetización ${ }^{8}$. En el caso de la escritura, inicialmente se planeó tratar temas como la estructura gramatical de la frase, los signos de puntuación y la ortografía. Además, en los últimos niveles del programa se planeó practicar la lectura y la escritura alrededor de cuentos y noticias de periódicos.

Con respecto a este último punto, cuando el investigador le pidió a los estudiantes escribir cuentos, sucedió que ellos quedaron bloqueados y una tensión se apoderó del salón porque no se les ocurría qué podían escribir usando ese género. Algunas personas lo intentaron, pero era notorio que el ejercicio no tenía sentido para ellas. Lo mismo pasó con la lectura de periódicos. Clases más adelante el investigador, basado en la perspectiva relativista, les pidió a los estudiantes que escribieran historias de espantos o espíritus de las que tuvieran conocimiento directo o indirecto, porque así podían aplicar lo aprendido a las experiencias propias de sus contextos locales. Efectivamente, la tensión que

6 Estos resultados pertenecen al trabajo de grado de Andrey Castiblanco (2017) para el departamento de Antropología de la Pontificia Universidad Javeriana.

7 Castiblanco es profesor de lectoescritura del programa desde 2015 y desde 2016 es líder de lectoescritura.

8 Los pénsums son diseñados por la Coordinación de Alfabetización de ColombiaCrece, que pertenece a la Dirección de Educación de la fundación y se compone de un coordinador y un líder para cada materia. Los voluntarios de la Coordinación suelen ser las personas que llevan más tiempo en el programa. 
hubo cuando se les pidió escribir cuentos no apareció y, por el contrario, todas las personas hablaron de historias de sus pueblos y la escritura fluyó. A partir del registro de eventos de lectura y escritura como estos, los contenidos del programa se ajustaron un poco más a las prácticas de los estudiantes: ya no se leen noticias ni se escriben cuentos; en su lugar, se usan contenidos basados en las experiencias previas de aquellos, como historias de espantos, autobiografías, recetas de cocina o cartas.

Otro cambio que se observó durante la investigación estuvo relacionado con la corrección de ortografía. Los estudiantes manifestaron haber sido maltratados en el pasado por no saber leer ni escribir. Cuando el investigador empezaba a corregir los errores ortográficos, los estudiantes asumían que lo que escribían estaba mal y que no sabían escribir, en parte porque la explicación que se suele dar cuando se corrige un error de ese tipo es que la palabra está mal escrita. Esto le hace pensar al estudiante que, efectivamente, no está aprendiendo a escribir cuando, en realidad, es algo que no puede saber de antemano ${ }^{9}$. Corregir insistentemente la ortografía hacía que los estudiantes se sintieran inseguros de lo que escribían y preguntaran reiteradamente si estaban escribiendo bien o mal. Por ejemplo, en un evento de lectura y escritura observado, una beneficiaria del programa (que decía que, como ella no sabía leer ni escribir, no sabía nada) preguntó al final de un ejercicio si estaba bien lo que había escrito. Cuando el investigador le corrigió la ortografía, ella dijo: “QQué pena! Es que yo no aprendo”, y borró apresuradamente y avergonzada todo lo que había escrito. Basándose en estas observaciones el investigador decidió dejar de hacer corrección ortográfica, con lo que disminuyó perceptiblemente la inseguridad de los estudiantes y, en consecuencia, facilitó la escritura de textos. Sin embargo, los textos resultantes se desviaron significativamente de la norma ortográfica establecida.

Desde una perspectiva relativista, la importancia de producir textos que sigan las normas ortográficas varía según el contexto social y cultural de las personas, sus necesidades y expectativas. En el programa había dos grupos que estaban interesados por distintas razones en la lectura y la escritura. El primer grupo, que era el mayoritario, estaba conformado principalmente por adultos mayores provenientes de zonas rurales del país, que en su mayoría ya no trabajaban ni querían terminar la escuela secundaria. Estaban interesados en lo que puede llamarse alfabetización funcional: querían leer el cartel del bus, firmar, leer la Biblia, escribir cartas a los nietos, etc. ${ }^{10}$ Para ellos, la ortografía no era una necesidad apremiante. El segundo grupo estaba conformado principalmente por personas

9 En ColombiaCrece, y en gran parte de Colombia, la escritura se enseña por medio de la asociación entre un grafo y su fonema. Sin embargo, los dialectos del español colombiano tienen el mismo fonema (una fricativa alveolar sorda) para las letras $s, z y c$ - esta última, cuando se combina con la e y la i-, así como tienen otro (una oclusión bilabial sonora) para la $b$ y la $v$. Por ello, cuando los estudiantes tienen que escribir cualquier palabra con estas letras se enfrentan a muchas posibilidades en las que solo una es la correcta. Por ejemplo, la palabra base, por sus fonemas, puede escribirse de seis formas distintas: base, bace, baze, vase, vace y vaze. A partir de la relación entre grafo y fonema, las probabilidades de que los estudiantes elijan la opción correcta son bajas.

10 Bernard Lahire muestra que la escuela impuso a la sociedad su concepción de lo que realmente es leer y escribir. Así, "la lectura entrecortada, discontinua, informativa, documental, rápida, técnica, etc., etc., a ojos de la mayor parte de los comentaristas-letrados, como también de quienes tienen esas prácticas de lectura, parece ser una 'infralectura', una 'sublectura' o una 'no lectura'” (2006, p. 159). 
que querían validar la escuela secundaria, por lo que consideraban necesaria la ortografía para obtener buenas calificaciones y, eventualmente, el título de bachiller, con el fin último de "salir adelante", esto es, conseguir mejores empleos, un mejor salario y una mejor calidad de vida.

Se podría argumentar que, cuando se decidió no corregir la ortografía, se aplicó un modelo relativista que pudo traer dificultades a los estudiantes que requerían esta habilidad para validar la secundaria. Efectivamente, escribir siguiendo las normas de ortografía es parte de los estándares requeridos para validar la educación básica en Colombia (Ministerio de Educación Nacional, MEN, 1998; 2006). Sin embargo, no es claro que corregir insistentemente la ortografía en los eventos de lectura y escritura que se observaron en el programa fuese una técnica que les permitiese a los estudiantes interesados desarrollar la habilidad de escribir siguiendo las normas ortográficas. En cambio, las modificaciones que se le hicieron a los contenidos del programa permitieron que se correspondiera un poco más con el contexto social y cultural de la mayor parte de los estudiantes, y facilitaron los eventos de escritura. No obstante, ya que la lectura y la escritura han sido concebidas largamente bajo el modelo universalista, las prácticas pedagógicas, los deseos de los estudiantes y las expectativas sociales están adecuadas a esta concepción.

En cuanto a las expectativas, los estudiantes manifestaron estar a gusto con la enseñanza mediante cartas, recetas de cocina y biografías, pero consideraron que en clase debería haber más dictados, lecturas en voz alta y planas. Además, aunque parezca paradójico, a pesar de que muchas de las personas dijeron que querían aprender para cumplir tareas cotidianas como comprar el mercado y tomar el bus, estas tareas las realizaban sin mayor dificultad (por ejemplo, sabían cuántos billetes costaba el mercado y preguntaban si el bus que venía les servía para ir a su lugar de destino). Así, muchas veces la motivación para entrar al programa era más simbólica que funcional (Rogers, 1999). Por ello, los estudiantes dieron como razones para aprender a leer y a escribir que así podrían hablar con personas alfabetizadas, dar ejemplo a los hijos, "ser alguien en la vida" o ilustrarse (las dos últimas son ejemplos claros de la perspectiva universalista).

En cuanto a la estructura social, el modelo universalista ha creado, de forma arbitraria, una serie de jerarquías que regulan el acceso a diferentes formas de participación (Farr, 2009). Así, antes solo quien sabía leer y escribir podía votar, y ahora se considera que solo quien sabe leer y escribir puede acceder a ciertos empleos. Por supuesto, es claro que leer y escribir es indispensable para muchos trabajos, pero según lo que contaron los estudiantes del programa esta idea se ha extendido hasta incluir empleos en los que no es indispensable. Este es el caso de una estudiante que aplicó a un trabajo como empleada en una casa donde debía cocinar y hacer el aseo: no consiguió el trabajo porque los empleadores pensaron que, dado que ella no sabía leer, podía confundir la sal con el azúcar. Así como ella, muchos de los estudiantes de ColombiaCrece necesitan saber leer y escribir porque solo así podrán trabajar en oficios como 
cuidar adultos mayores o cortar el pelo, en los que se exige la alfabetización, aunque se puedan aprender y ejercer sin el lenguaje escrito.

Nosotros consideramos que en el programa de alfabetización estuvieron presentes tanto la perspectiva relativista, que se aplicó para adecuar la lectura y la escritura al contexto de los participantes, como la perspectiva universalista, que existe en las estructuras educativas, las creencias que hay acerca de la alfabetización y las jerarquías que estas creencias han establecido. Ambas perspectivas conviven en el programa, no siempre de forma armoniosa.

\section{Ulular}

Ulular es un grupo informal de lectura y escritura que se reúne semanalmente en Guatavita, un pueblo cercano a Bogotá. El grupo nació en 2014, cuando dos profesores decidieron crearlo para aplicar sus propias concepciones acerca de cómo enseñar a leer y a escribir sin tener las limitaciones que les imponía la escuela. Los investigadores asistimos a la mayor parte de las sesiones durante un año y participamos activamente en las prácticas de lectura y escritura que se realizaron. Además de la observación participante, hicimos entrevistas sin estructurar tanto a los profesores como a los asistentes más permanentes.

Durante ese año (2015), al grupo asistieron en total unas doce personas, la mayor parte estudiantes de la escuela local y algunos jóvenes universitarios de Bogotá. El grupo se reunía en un café cerca del parque principal del pueblo. A cada sesión siempre asistieron los profesores y cuatro o cinco jóvenes. Algunos de los asistentes eran miembros más o menos permanentes, mientras que otros iban ocasionalmente.

Al empezar el grupo, los profesores orientaban los eventos de lectura y escritura mostrando a los estudiantes e investigadores textos literarios, videos musicales e imágenes artísticas, para después pedirles que escribieran textos cortos basados en lo que habían visto y escuchado. Más adelante, los jóvenes del grupo empezaron a orientar ellos mismos los eventos siguiendo ese mismo esquema. Por ejemplo, en una ocasión un joven pidió a los participantes leer un texto tomado de una revista acerca de sociedades secretas y teorías de la conspiración. El joven propuso un ejercicio de escritura en el que los participantes tenían que imaginar su propia sociedad secreta y escribir acerca de ella. El ejercicio generó un diálogo entre todos los participantes, en el que cada quien pudo mencionar textos, películas o anécdotas relacionadas con el tema.

Al finalizar el año, los integrantes de Ulular decidieron publicar una revista en papel y un blog en internet, basados en la producción escrita del grupo (Ulular, 2015a; 2015b). Los jóvenes del grupo consiguieron parte de los recursos para imprimir la revista vendiendo productos de aseo con poesías escritas por ellos mismos en las etiquetas. Con ayuda de los profesores, diagramaron e imprimieron todo el material. Los investigadores ayudamos tangencialmente 
con eso y con la creación del blog. Adicionalmente, organizamos un evento en Bogotá para presentar la revista al público (Instituto Caro y Cuervo, ICC, 2015).

Al principio, los investigadores creímos que Ulular estaba basado en la pedagogía crítica de Paulo Freire (1977), aunque no fuese nombrado explícitamente por los profesores. Nuestra suposición estuvo basada en que este autor es reconocido por criticar la educación tradicional (a la que califica de "bancaria") y proponer que el punto de partida de la educación debería ser la reflexión sobre el contexto cotidiano de los estudiantes, en lugar de unos planes de estudio definidos de antemano. Los investigadores creímos que una aplicación de estas ideas era el hecho de que en Ulular el punto de partida fueran textos provenientes de los medios masivos de comunicación y la industria cultural, de temas como las sociedades secretas, los relatos de terror o el esoterismo, porque estos hacían parte del contexto cotidiano de los estudiantes y eran valorados en contraste con los textos escolares. Al respecto, una joven dijo:

En el colegio [leer y escribir] es muy académico. Hay que leer para hacer un ensayo o un análisis [...]. En cambio, en Ulular uno puede hacer un escrito de algo muy común [...], puede ser recursivo, utilizar las cosas del ambiente. (P. Castillo, comunicación personal, 16 de mayo de 2016)

Por otra parte, la pedagogía propuesta por Freire tiene como meta generar conciencia y pensamiento crítico a partir de la reflexión y el diálogo acerca del ambiente de los estudiantes. Para lograr esto, se requiere que la relación entre estos y los profesores sea horizontal y que los estudiantes se apropien de su proceso educativo. Que los jóvenes participaran voluntariamente en Ulular, que dirigieran ellos mismos los eventos de lectura y escritura y que en cada uno de estos hubiera diálogo entre todos los participantes, no condicionado por un plan de estudios predeterminado, nos parecieron indicios fiables de una aplicación de las ideas de Freire.

Adicionalmente, en Ulular se partía del supuesto de que leer y escribir tenían efectos culturales y cognitivos particulares. Según Lesley Bartlett, esto hace parte de las ideas pedagógicas de Freire:

Gran parte del trabajo en alfabetización crítica [(la pedagogía de Freire)] mantiene lo que Street llama ideologías "autónomas" sobre las consecuencias de leer y escribir, que exageran o simplifican en exceso los efectos de la alfabetización. Por ejemplo, la discusión de Freire acerca de la alfabetización como una herramienta para la liberación sugiere una consecuencia autónoma de la lectura y la escritura. (Bartlett et al., 2011, p. 160, traducción nuestra) ${ }^{11}$

11 En el original: "Yet much of the work in critical literacy maintains what Street called "autonomous" ideologies about the consequences of literacy, which overstate and oversimplify literacy's effects. For example, Freire's discussion of literacy as a tool for liberation suggests an autonomous consequence to literacy". 
En efecto, para Freire aprender a leer y a escribir está estrechamente relacionado con la posibilidad de tener pensamiento crítico, lo cual es una perspectiva muy semejante a la que se tiene en Ulular.

Sin embargo, cuando les compartimos nuestra interpretación a los profesores, se sorprendieron, puesto que para ellos Ulular no estuvo basado en la aplicación de las ideas de ese autor. El club no se basó en unos modelos teóricos específicos ni en un diseño organizativo particular. Más bien, fue el fruto de las conversaciones entre los profesores y estuvo basado en sus experiencias y aspiraciones. Una de las profesoras comentó:

Siempre he pensado que [Ulular] se mueve con otra clase de principios. Es más, ni siquiera pedagógicos, porque no hay una intención pedagógica, aunque se haga la pedagogía. Mi intención no es hacer un aula de clases acá. Paulo Freire lo pensaba de otra manera. (M. Guerrero, comunicación personal, 5 de abril de 2017)

Para el otro profesor "podría [en cambio] hablarse de aprendizaje dialógico para complementar la ideas de Freire, e incluso hablar un poco de inteligencia cultural cuando se habla del trabajo que hacen los chicos al realizar las sesiones" (A. Cruz, comunicación personal, 2 de abril de 2017). En efecto, Nicholas Burbules (1999) sostiene que el diálogo en la enseñanza debe ser entendido como una relación social en la que hay conciencia del acto comunicativo y se fomenta el reconocimiento del otro y de uno mismo, en lugar de entenderlo como un método para conseguir un consenso, un acuerdo, un hallazgo o un ganador. Según el autor, el diálogo

no es algo que hagamos o que empleemos; es una relación en la que entramos: a veces atrapados, a veces, llevados por ella. Considerar el dialogo como una relación (con otra persona o con otras personas) destaca en él aspectos que están más allá de nosotros, que descubrimos y que nos modifican. (Burbules, 1999, p. 15)

Los investigadores creemos que en Ulular el diálogo ocurría de esta manera porque el grupo no se encontraba en el contexto de un salón de clases, en el que la conversación la orienta el profesor basado en un plan de estudios con intenciones pedagógicas fijadas antemano. En cambio, el diálogo ocurría en espacios fuera de la escuela, en donde las relaciones entre profesores y estudiantes eran más horizontales y los objetivos estaban sin fijar. Oralidad y escritura ocurrían al mismo tiempo. Desde nuestro punto de vista, esto es semejante a lo que identificó Heath en las comunidades estadounidenses que observó, en las que la oralidad y la escritura no estaban separadas (2009, ver arriba). Así, sostenemos que la posición que tuvo Ulular acerca de la escritura tomó distancia de la perspectiva universalista que, como vimos, concebía a la escritura como algo fundamentalmente separado de la oralidad. 
Finalmente, hubo un aspecto fundamental de la pedagogía de Freire del cual los profesores de Ulular se distanciaron. En Freire el proceso de concientización que produce la educación debe tener propósitos emancipatorios y revolucionarios, mientras que los profesores de Ulular no suscribieron estos propósitos. En la revista, uno de ellos escribió:

Toda manera de trascender ética, política y filosófica de esta revista es pura coincidencia. Si decimos sublevar, es contra los que dormitan, [...] los burócratas, $[. .$.$] nuestras madrespadres, [\ldots]$ los choferes de autobús y [...] quienes escriben desde su Olimpo imaginario. Practicamos un acto de rebeldía en la lectura y la escritura; no crea que estamos acá para tumbar ídolos, mandarnos de curas a guerrilleros o alguna vaina así. Eso ya se lo inventaron unos locos-mechudos que ahora están calvos o muertos. (Cruz, 2015)

El hecho de tomar distancia de este tipo de propósitos no significaba que Ulular careciese de objetivos, pero sí implicaba que estos eran implícitos y no explícitos. $\mathrm{Al}$ respecto, uno de los profesores comentó que, en retrospectiva, Ulular tenía la intención de transformar

las estructuras de pensamiento de los participantes más fieles. Incluso me arriesgaría a proponer la idea de crear una ciudadanía volcada hacia un amor por lo académico y estético. Es decir, el proyecto surgió con el objetivo implícito de crear un tipo de sujeto particular. (A. Cruz, comunicación personal, 2 de abril de 2017)

En definitiva, desde nuestro punto de vista en Ulular aparecían al mismo tiempo la perspectiva relativista, en cuanto que se tenían en cuenta las preferencias de los estudiantes y no se separaba tajantemente la oralidad y la escritura (enfoque dialógico), y la perspectiva universalista, en cuanto que se le atribuía a la escritura el poder de generar pensamiento crítico, aunque esto se matizó cuando los profesores se separaron de los propósitos emancipatorios y revolucionarios de la pedagogía crítica de Freire.

\section{Conclusiones}

En este artículo vimos que los estudios antropológicos sobre lectura y escritura partieron de una concepción inicial en la cual se suponía que el lenguaje escrito producía en sí mismo una transformación cultural y cognitiva, que tendía hacia el pensamiento crítico y abstracto, en particular cuando estaba relacionado con ciertas formas propias de la tradición occidental. A estas concepciones se las llamó modelo autónomo, pero podríamos también denominarlas perspectivas universalistas. 
Vimos cómo la evidencia etnográfica ha puesto en duda estas perspectivas al demostrar que la lectura y la escritura no necesariamente producen los efectos que se esperaban. Además, algunos antropólogos han señalado que las perspectivas universalistas han producido discriminación en contra de las formas de conocimiento que no están ancladas en lo escrito, y hacen invisibles prácticas de lectura y escritura que tienen formas diferentes a las de la tradición occidental. Para estos antropólogos hay tantas formas de leer y escribir como diversos grupos sociales hay en el mundo. Parten de una concepción que se ha llamado modelo ideológico y que nosotros hemos denominado perspectiva relativista. Hemos señalado también que, desde esta perspectiva, leer y escribir tienen sentido en eventos particulares (literacy events).

Para los estudiantes del programa de alfabetización de ColombiaCrece, que provenían mayoritariamente de zonas rurales, la alfabetización se facilitó cuando estuvo basada en narrativas no ancladas en lo escrito, como los relatos de espantos o espíritus, mientras que formas propias de la tradición occidental, como los cuentos y las noticias de periódico, no resultaron tan pertinentes (perspectiva relativista). En ese mismo programa, la producción de textos se facilitó al no corregir la ortografía. Al respecto, argumentamos que la producción de textos que se desvía de la norma ortográfica no necesariamente era un problema para los estudiantes de mayor edad, que en su contexto local solo requerían una escritura funcional, pero que sí lo era para los estudiantes más jóvenes, porque leer y escribir normativamente bien es un requisito para validar la secundaria, que a su vez es requerida para acceder a puestos de trabajo deseados (perspectiva universalista). Además, constatamos que las expectativas y motivaciones de los estudiantes, así como las estructuras sociales en las que están inmersos, muchas veces responden a una perspectiva universalista.

Para los estudiantes del club Ulular, la lectura y la escritura se facilitaron por la inclusión de temas provenientes de los medios masivos de comunicación y por el énfasis dado a las relaciones entre oralidad y escritura, que permitía la aplicación de una pedagogía basada en el diálogo, entendido como una relación social (perspectiva relativista). Al mismo tiempo, a la escritura se le atribuía el efecto de producir pensamiento crítico (perspectiva universalista), pero no con propósitos emancipatorios y revolucionarios, como lo plantearía la pedagogía crítica de Freire.

En ambos casos vimos cómo las perspectivas universalistas y relativistas coexisten en la práctica. En el caso de ColombiaCrece vimos cómo conviven prácticas en las que se reconoce el contexto de los participantes, con expectativas como que leer y escribir permite a los estudiantes "ser alguien en la vida" e ilustrarse o ideas como que en las clases debería haber más planas y dictados. En el caso de Ulular, vimos cómo convive la inclusión de elementos del contexto en prácticas dialógicas que combinan la oralidad y la escritura, con la idea de que la escritura produce en sí misma pensamiento crítico, aunque no con fines emancipatorios ni revolucionarios. 


\section{$\mathbf{R}_{\text {eferencias }}$}

Bartlett, L., López, D., Vasudevan, L. y Warriner, D. (2011). The anthropology of literacy. En B. Levinson y M. Pollock (eds.), A companion to the anthropology of education (pp. 154-176). Chichester: Wiley-Blackwell.

Barton, D., y Hamilton, M. (2000). Literacy practices. En D. Barton, M. Hamilton y R. Ivanič (eds.), Situated literacies: Reading and writing in context (pp. 7-14). Londres: Routledge.

Besnier, N. (1988). The linguistic relationships of spoken and written Nukulaelae registers. Language, 64(4), 707-736.

Burbules, N. (1999). El diálogo en la enseñanza: teoría y práctica. Buenos Aires: Amorrortu.

Castiblanco, A. (2017). "Como no sabe leer ni escribir, tratémosla mal". Análisis de ideologías de la alfabetización en el sector El Codito de Bogotá (Trabajo de grado inédito). Pontificia Universidad Javeriana, Bogotá.

Conklin, H. (1949). Bamboo literacy on Mindoro. Pacific Discovery, 3, 4-11.

Cruz, A. (2015, diciembre 1). Editorial: unísono nocturno. Revista Ulular, 1, 1-2.

Farr, M. (2009). Ideologías de la alfabetización: prácticas locales y definiciones culturales. En J. Kalman y B. Street (eds.), Lectura, escritura y matemáticas como prácticas sociales: diálogos con América Latina (pp. 99-111). México: Siglo XXI-Crefal.

Ferguson, C. (1987). Literacy in a hunting-gathering society: The case of the Diyari. Journal of Anthropological Research, 43(3), 223-237.

Freire, P. (1977). Pedagogía del oprimido. Montevideo: Siglo XXI.

Goody, J. (2000). The power of the written tradition. Washington D. C.: Smithsonian Institution Press.

Goody, J. y Watt, I. (1996). Las consecuencias de la cultura escrita. En J. Goody (ed.), Cultura escrita en sociedades tradicionales (pp. 3-82). Barcelona: Gedisa.

Heath, S. B. (2009). Ways with words: Language, life, and work in communities and classrooms. Nueva York: Cambridge University Press.

Hymes, D. (1986). Models of the interaction of language and social life. En J. Gumperz y D. Hymes (eds.), Directions in sociolinguistics: The ethnography of communication (pp. 35-71). Nueva York: Blackwell. 
Instituto Caro y Cuervo (ICC) (2015, diciembre 1). Viajes de la escritura: la experiencia de Ulular. Recuperado de https://www.youtube.com/ watch? $=$ ukHDX-HDHYI\&t=9m10s

Kalman, J. (2003). Escribir en la plaza. México: Fondo de Cultura Económica.

Lahire, B. (2006). Lógicas prácticas: el "hacer" y el "decir sobre el hacer". En El espíritu sociológico (pp. 137-155). Buenos Aires: Manantial.

Luke, A. y Freebody, P. (1997). Shaping the social practices of reading. En S. Muspratt, A. Luke y P. Freebody, Constructing critical literacies: Teaching and learning textual practice (pp. 185-226). Creskill, NJ: Hampton Press.

Maddox, B. (2005). Assessing the impact of women's literacies in Bangladesh: An ethnographic inquiry. International Journal of Educational Development, 25, 123-132. DOI: https://doi.org/doi: 10.1016/j.ijedudev.2004.11.017

Marinho, M. (2009). Nuevas alfabetizaciones en los procesos sociales de inclusión y exclusión. En J. Kalman y B. Street (eds.), Lectura, escritura y matemáticas como prácticas sociales: diálogos con América Latina (pp. 40-63). México: Siglo XXI-Crefal.

Ministerio de Educación Nacional (MEN) (ed.) (1998). Lineamientos curriculares de la lengua castellana. Bogotá: MEN.

Ministerio de Educación Nacional (MEN) (ed.) (2006). Estándares básicos de competencias en lenguaje, matemáticas, ciencias y ciudadanas. Bogotá: MEN.

Ong, W. J. (1987). Oralidad y escritura: tecnologías de la palabra. México: Fondo de Cultura Económica.

Papen, U. (2001). "Literacy-Your key to a better future" ?: Literacy, reconciliation and development in the National Literacy Programme in Namibia. En B. Street (ed.), Literacy and development: Ethnographic perspectives (pp. 40-60). Londres: Routledge.

Papen, U. (2005). Literacy and development: What works for whom? Or, how relevant is the social practices view of literacy for literacy education in developing countries? International Journal of Educational Development, 25(1), 5-17. DOI: https://doi.org/10.1016/j.ijedudev.2004.05.001

Roberts, C. y Street, B. (2013). El lenguaje escrito y hablado. En F. Coulmas (ed.), Manual de sociolingüística (pp. 187-205). Bogotá: Instituto Caro y Cuervo.

Rogers, A. (1999). Improving the quality of adult literacy programmes in developing countries: The "real literacies" approach. International Journal of Educational Development, 19(3), 219-234. DOI: https://doi.org/10.1016/ S0738-0593(99)00015-2 
Street, B. (1993). Introduction: The new literacy studies. En B. Street (ed.), Crosscultural approaches to literacy (pp. 1-22). Nueva York: Cambridge University Press.

Street, B. (2009). Perspectivas etnográficas y políticas sobre cultura escrita: el poder de nombrar y definir. En J. Kalman y B. Street (eds.), Lectura, escritura y matemáticas como prácticas sociales: diálogos con América Latina (pp. 84-98). México: Siglo XXI-Crefal.

Street, B. (2013). What's "new" in new literacy studies? Critical approaches to literacy in theory and practice. Current Issues in Comparative Education, 5(2), 77-91.

Street, B., Rogers, A. y Baker, D. (2006). Adult teachers as researchers: Ethnographic approaches to numeracy and literacy as social practices in South Asia. Convergence, 39(1), 31-44.

Ulular (2015a). Blog Ulular. Recuperado de https://revistaulular.wordpress.com/

Ulular (2015b). Revista Ulular. Recuperado de https://revistaulular.wordpress. com/

Unesco (2005). Education for all. Global monitoring report 2006: Literacy for life. París: Unesco. Recuperado de http://unesdoc.unesco.org/ images/0014/001416/141639e.pdf

Velasco, H. y Díaz de Rada, Á. (2006). La lógica de la investigación etnográfica: un modelo de trabajo para etnógrafos de la escuela. Madrid: Trotta.

Wickens, C. y Sandlin, J. (2007). Literacy for what? Literacy for whom? The politics of literacy education and neocolonialism Unesco - and World Bank - sponsored literacy programs. Adult Education Quarterly, 57(4), 275292. DOI: https://doi.org/10.1177/074173607 